\title{
Peaceful Utilization of Nuclear Energy in Iran: Detection of Sentinel Node in Breast Cancer Patients using Technetium Element
}

\author{
Masoud Moslehi1*
}

Ahmad Shanei ${ }^{1}$

Golshan Mahmoudi2

\begin{abstract}
${ }^{1}$ Department of Medical Physics and Medical Engineering, School of Medicine, Isfahan University of Medical Sciences, Isfahan, Iran, Email: mmoslehi_m@yahoo.com ${ }^{2}$ Department of Medical Physics, Sabzevar University of Medical Sciences, Sabzevar, Iran
\end{abstract}

\section{Doi:10.5901/mjss.2015.v6n1s1p643}

\begin{abstract}
Iran has had a peaceful nuclear program for more than 50 years, beginning with a research reactor purchased from the United States in 1959. There were a few suspicions of a nuclear weapons program, but these strongly abated in the decades after Iranian revolution in 1979. One of the most useful applications of nuclear energy in Iran is radionuclide imaging in sentinel lymph detection of breast cancer using Technetium (TC) element. Sentinel lymph node is the first regional lymph node that drains the lymph from the primary tumor. It is potentially the first node to receive the seeding of lymph-borne metastatic cells. For sentinel node detection, one of the methods most often used in Iran is radionuclide imaging. However, guidelines strongly recommend imaging of the sentinel node combined with identification with a gamma probe before any operative procedure, rather than the use of external probe detection alone. In this regard, guidelines do not provide defined protocols for image acquisition and there is much controversy about the usefulness of dynamic imaging. This study aimed to discuss procedural guidelines for Lymphoscintigraphy for detection of sentinel node using 99mTc-Phytate in Isfahan, Iran.
\end{abstract}

Keywords: Nuclear Energy, Breast cancer, ${ }^{99 m}$ Tc-Phytate, Lymphoscintigraphy, Iran

\section{Introduction}

Iran has had a peaceful nuclear program for more than 50 years, beginning with a research reactor purchased from the United States in 1959. The Iran first plan to build 23 nuclear power reactors by the 1990s was regarded as grandiose, but not necessarily viewed as a "back door" to a nuclear weapons program, possibly because Iran did not then seek the technologies to enrich or reprocess its own fuel. There were a few suspicions of a nuclear weapons program, but these strongly abated in the decades after Iranian revolution [1]. One of the most useful applications of nuclear energy in Iran is radionuclide imaging in detection of breast cancer using Technetium (Tc) element. In this regard, sentinel lymph node is the first regional lymph node that drains the lymph from the primary tumor. It is potentially the first node to receive the seeding of lymph-borne metastatic cells [2]. The presence or absence of metastasis to locoregional lymph nodes, especially axillary nodes, has major prognostic and therapeutic implications for patients with breast cancer [3-5]. There is ample evidence that a tumor negative sentinel lymph node is a reliable predictor for the absence of tumor invasion in other lymph nodes $[3,6]$.

Lymphoscintigraphy [7] allows the surgeon to easily identify and biopsy the sentinel lymph node. This method identifies the sentinel node but cannot determine if it is involved with cancer [8].

There are different approaches; however, guidelines strongly recommend imaging of the sentinel node combined with identification with a gamma probe before any operative procedure, rather than the use of external probe detection alone. The first 'hot spot' detected on images has to be considered the sentinel node [9-11]. Imaging also offers the ability to identify different pathways of lymphatic drainage from the tumor, resulting in the detection of more and extra-axillary sentinel nodes [12].

Generally it is stated that static images should be taken hours after injection (2-18 h) [10]. The Society of Nuclear Medicine has no specific guidelines on sentinel node procedures.

Because of the low visualization rate of sentinel nodes on dynamic imaging during the 30 min after injection, we 
changed our protocol [13-16]. In March 2013 we started our current protocol, in which static images are acquired 5, 30 and 120 min after injection. This study aimed to discuss procedural guidelines for Lymphoscintigraphy for detection of sentinel node using 99mTc-Phytate in Isfahan, Iran.

\section{Procedural Guidelines}

General guidelines are in accordance with those previously reported by EANM [10]. EANM has written and approved these guidelines to promote the cost-effective use of high-quality nuclear medicine procedures. Therefore in this section, the approved guidelines by EANM are presented accordingly [10].

A variety of colloids have been used in this technique. The radiopharmaceuticals commonly employed for LS are ${ }^{99 m} T c-s u l p h u r$ colloid (particles' size: $15-5,000 \mathrm{~nm}$ ), ${ }^{99 \mathrm{~m} T c-n a n o c o l l o i d ~(5-100 ~ n m), ~}{ }^{99 \mathrm{~m} T c-a n t i m o n y}$ trisulphide (3-30 nm). The choice of the radiopharmaceutical depends on the geographic area: in Europe, $99 \mathrm{mTC}$-nanocolloid; in USA, $99 \mathrm{mTC}$ -

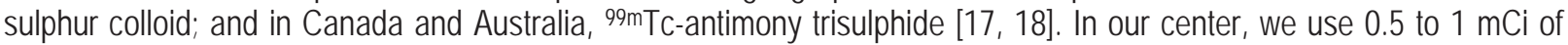
99mTc-Phytate.

There is a general agreement that a radiocolloid with the majority of particles ranging between 100 and $200 \mathrm{~nm}$ in size can be considered the best compromise between fast lymphatic drainage and optimal retention in the sentinel lymph node $[17,18]$.

If a single node wishes to be seen and imaging times cannot be coordinated with the operating theatre time, a large colloid with a size of $200-1,000 \mathrm{~nm}$ is recommended $[7,9,19]$. It has been found that these larger colloids tend to "stick" in the sentinel node and allow imaging for up to $20 \mathrm{~h}$ post injection.

The SN is, generally, visualized in $2 \mathrm{~h}$, and the patient should be in the operating theatre within about $16-20 \mathrm{~h}$ after the injection of the colloid $[2,5,8,11,18]$. The colloid must be labelled with technetium pertechnetate using manufacturer's instructions. A labelling yield greater than $95 \%$ must be assessed before injecting the radiopharmaceutical. General radiopharmaceutical requirements for quality control must be used $[18,20]$.

Large volumes of colloid may disrupt local lymphatics; therefore, small volumes should be injected [8, 21]. A single aliquot of $5-20 \mathrm{MBq}$ (depending on the elapsed time between scintigraphy and surgery) of colloid in $0.2 \mathrm{ml}$ is considered sufficient. A higher activity can be used for late procedures. The syringe should also contain a similar amount of air to clear any dead space within the syringe and the needle. In deep lesions, a slightly larger volume $(0.5 \mathrm{ml})$ may be used.

Two type of injection are widely used for this method. A sub-dermal injection over the tumor site is sufficient for all but the deepest tumors $[8,21]$. The site of injection can be gently massaged after the administration or if passage of activity from the injection site is delayed at any time during the study. A peri-tumoral injection of $0.5 \mathrm{ml}$ is recommended in all deep tumors [21, 22]. If the lump is not palpable, ultrasound can be used to guide the injection.

Peri-areolar injection can be used particularly in upperquadrant tumors to avoid possible cross-talk due to short distance between peri-tumoral depot and the axillary sentinel node $[22,23]$. This technique has the advantage of demanding less experience, particularly in non-palpable lesions. At present there is no evidence to justify intratumoral injections of colloids.

Imaging is strongly recommended before any operative procedure $[24,25]$, as there is some variability in breast lymphatic drainage into the axilla and more than one sentinel node can be visualised in up to $20 \%$ of patients.

\section{Lymphoscintigraphy for Detection of Sentinel Node in our Center}

Lymphoscintigraphy for detection of sentinel node in our center is included intra-dermal injection of 0.5 to $1 \mathrm{mCi}$ of $99 \mathrm{mTC}$ Phytate. Then, 5, 30 and 120 minute hands up lymphoscintigraphy is performed. All images in this center are acquired using Gamma camera (energy $140 \mathrm{keV}$, window 15-20\% and LEHR collimator). For all patients, the scintigraphic images are retrospectively evaluated. All hot spots are noted and classified according to anatomic location and designated as sentinel node or higher echelon node.

The evaluation is carried out by one nuclear medicine physician and one trainee in nuclear medicine, both experienced in sentinel node procedures. Where there are different interpretations, a consensus will be found [26]. All surgical and pathologic reports are retrospectively screened to score the number of nodes removed, the histology of the primary tumor, the presence of malignant cells in the sentinel node, the number of axillary lymph node dissections (ALND) and to determine whether malignancy was present in the nodes after ALND.

Report of the first year's clinical experience of 99mTc-Phytate lymphoscintigraphy for detection of sentinel node using the above mentioned protocol is under preparation.

The gamma camera used for lymphoscintigraphy in our center is equipped with a low-energy, high-resolution 
collimator. The energy window is $15 \%$ ( $\pm 5 \%$ ) centered over the $140 \mathrm{keV}$ photopeak of $99 \mathrm{mTc}$.

To position the patient, the patient lies supine with hands up for imaging on the gamma-camera bed. Anterior and

$45^{\circ}$ anterior oblique imaging should be obtained. It is useful if the arm on the side of the cancer is extended laterally to $90^{\circ}$, as this will be the position during surgery.

In this protocol, truncation of the high-activities (injection site) will improve visualization of the sentinel node. A logarithmic scale to enhance low-count areas instead of a linear scale is preferable for image display [7].

The rest of process of this protocol is listed as bellow:

\subsection{Reporting}

The report to the referring physician should describe:

- Site of image acquisition (projections of breast and axilla)

- Radiopharmaceutical, way of administration, and amount of activity injected

- Location of the sentinel node(s) on gamma-camera images

- Any source of error or inaccuracy of the procedure

\subsection{Sources of error}

As the rate of passage of the smaller colloids is variable, it is advisable that frequent or continuous measurement is performed to identify when activity has reached the sentinel node and determine when intra-operative probing will be optimal.

\subsection{Issues requiring further clarification}

- Even if some authors consider that the previous breast biopsy can alter the lymphatic drainage affecting the results of sentinel node biopsy, there are other evidences that sentinel node biopsy performed in the area of previous breast biopsy do not affect the accuracy of the procedure $[4,27]$.

- There is still an open discussion on the influence of neo-adjuvant chemotherapy on sentinel node identification and removal $[4,27]$.

- Multiple foci of carcinoma, particularly when located in different quadrants, have been considered a relative contraindication for sentinel lymph node biopsy because of concerns that these tumors involve more than one lymphatic vessel. Recent studies report that sentinel node localisation maintain its negative predictive value also in patients with multicentric and multifocal breast carcinoma [7, 18, 22].

- Though there is some evidence that peri-areolar injection will be effective in patients with suspected multifocal or multicentric lesions, this must be confirmed by larger trials [27, 28].

- There is no definitive consensus on what to do if the sentinel node cannot be visualized.

\section{Discussion}

Iran started peaceful utilization of nuclear energy many years ago. In this regard, Iran has asserted repeatedly that its nuclear program is strictly peaceful, stating in May 2003 that "we consider the acquiring, development and use of nuclear weapons inhuman, immoral, illegal and against our basic principles. They have no place in Iran's defense doctrine. [29]" Iranian officials have also insisted on their right to develop peaceful uses of nuclear technology. Former President Khatami stated in March 2005 that ending Iran's uranium enrichment program is "completely unacceptable," but that Iran would provide "objective guarantees" of the peaceful uses of enrichment. Nuclear energy can be used for both peaceful (medical applications) and military (nuclear weapons) uses. In Iran, one of the most useful applications of nuclear energy is radionuclide imaging in sentinel node detection of breast cancer using Technetium (Tc) element.

According to the definition, the sentinel node is the first draining lymph node on the direct pathway from the primary tumor site [27, 28]. This is a good argument for dynamic imaging directly after injection because it provides the possibility of detecting the 'real' sentinel node. However, this definition is not always applicable during surgery for practical considerations. Therefore, guidelines usually provide more practical definitions for pointing out the sentinel node with the gamma probe during surgery. The EANM guideline proposes that the surgeon must locate the lymph node with the highest activity guided by the skin mark based on the scintigraphic images and the gamma probe. When there are two or 
more of such lymph nodes, all should be removed [17, 23].

The Dutch guideline states that, after excision of the most active lymph node, the wound should be measured for high residual activity. If this activity exceeds $10 \%$ of the total activity in the removed lymph node, the surgeon must search for other active nodes and these must be removed as well [10,19]. Both of those recommendations do not take into account the anatomic basis of the sentinel node principle. Because of this discrepancy between theoretical and practical definitions, a less active node, first draining on the pathway from the tumor, may be left in situ, thereby strongly reducing the added value of dynamic imaging directly after injection.

The EANM guidelines give no strong recommendations for the site of injection, and the volumes suggested differ depending on the site of injection. There are three major differences in our protocol compared with the EANM guidelines: we administered a radioactive dose of 0.5 to $1 \mathrm{mCi}$ and in all the patients, after intra-dermal injection of 0.5 to $1 \mathrm{mCi}$ of 99mTc-Phytate, 5, 30 and 120 minute hands up lymphoscintigraphy was performed.

There is no assumption that a relatively high activity would have effect on the time point of sentinel node visualization. It has been suggested that administration of a larger quantity of nanocolloid would result in higher extractions from the injected site and higher accumulations in the lymph nodes [11, 18, 21, 22]. In some centers the most active node and the node closest to the tumor in the axillary region and the parasternal region are classified as sentinel nodes $[10,19]$. When lymph vessels are visible, the first node in the chain and the most active node are called sentinel nodes.

Considering optimal patient care [30], one could propose scanning every patient $30 \mathrm{~min}$ after injection, allowing the patient to be operated upon faster. In our experience operating schedules allow the patient to be operated upon earlier in the day, except for the first patient of the day to undergo surgery. However, in certain circumstances it may be helpful to speed up the procedures at the nuclear medicine department, considering a chance of visualization in 5 min.

\section{Conclusion}

In this study, procedural guidelines for Lymphoscintigraphy detection of sentinel node using ${ }^{99 m}$ Tc-Phytate in Iran were thought. Compared to other centers, guidelines provide defined protocols for image acquisition.

\section{Acknowledgement}

The authors wish to acknowledge the staff in Nuclear Medicine, Chamran Hospital, Isfahan University of Medical Sciences, Isfahan, Iran for their contribution to this study.

\section{References}

1. Statement by Iran's former Foreign Minister Kamal Kharrazi at [http://www.pbs.org/newshour/bb/middle_east/july-dec04/iran_927.html].

2. Keshtgar MR, Ell PJ. Sentinel lymph node detection and imaging. European journal of nuclear medicine. 1999;26(1):57-67.

3. Giuliano AE, Haigh PI, Brennan MB, Hansen NM, Kelley MC, Ye W, et al. Prospective observational study of sentinel lymphadenectomy without further axillary dissection in patients with sentinel node-negative breast cancer. Journal of clinical oncology : official journal of the American Society of Clinical Oncology. 2000;18(13):2553-9.

4. Turner RR, Ollila DW, Krasne DL, Giuliano AE. Histopathologic validation of the sentinel lymph node hypothesis for breast carcinoma. Annals of surgery. 1997;226(3):271-6; discussion 76-8.

5. Veronesi U, Paganelli G, Galimberti V, Viale G, Zurrida S, Bedoni M, et al. Sentinel-node biopsy to avoid axillary dissection in breast cancer with clinically negative lymph-nodes. Lancet. 1997;349(9069):1864-7.

6. Ali Alavian-Ghavanini, Aminollah Bahaodini, Ehsan Salimi. The effect of whole body gamma irradiation on nitric oxide pathway of rat's aorta. J Radiobiol. 2014;1(1):9-13.

7. Kumar R, Jana S, Heiba SI, Dakhel M, Axelrod D, Siegel B, et al. Retrospective analysis of sentinel node localization in multifocal, multicentric, palpable, or nonpalpable breast cancer. Journal of nuclear medicine : official publication, Society of Nuclear Medicine. 2003;44(1):7-10.

8. Veronesi U, Paganelli G, Viale G, Luini A, Zurrida S, Galimberti V, et al. A randomized comparison of sentinel-node biopsy with routine axillary dissection in breast cancer. The New England journal of medicine. 2003;349(6):546-53.

9. Baradaran-Ghahfarokhi M. Normal tissue complication probability modeling of radiation-induced bladder complications. J Radiobiol. 2014;1(1):19-20.

10. Buscombe J, Paganelli G, Burak ZE, Waddington W, Maublant J, Prats E, et al. Sentinel node in breast cancer procedural guidelines. European journal of nuclear medicine and molecular imaging. 2007;34(12):2154-9.

11. Morton DL, Bostick PJ. Will the true sentinel node please stand? Annals of surgical oncology. 1999;6(1):12-4. 
12. Lotfi M, Bagheri MH, Mosleh-Shirazi MA, Faghihi R, Baradaran-Ghahfarokhi M. Evaluation of the changes in the shape and location of the prostate and pelvic organs due to bladder filling and rectal distension. Iranian Red Crescent medical journal. 2011;13(88):51-60.

13. Moslehi M, Assadi M. Bilateral radioiodine uptake by the non-lactating breast of a single nulliparous woman: A case report and literature review. Archives of Medical Science. 2012;8(3):575-77.

14. Moslehi M, Assadi M. Contribution of ultrasound examination in the detection of neck recurrence in low-risk differentiated thyroid carcinoma patients at first follow-up visits. Nuclear Medicine Review. 2014;17(1):3-6.

15. Moslehi M, Rahimi M, Moradi-Khaniabadi B, Shahbazi-Gahrouei D. The effect of neck physical examination and signing thyroid nodules by lead marker on 99mTcO4 thyroid scan results. Journal of Isfahan Medical School. 2014;31(259).

16. Shahbazi-Gahrouei D, Bonyadi $P$, Moslehi M, Shahi Z. Effects of early liothyronine consumption after radioiodine therapy on accumulated dose and exposure rate in patients with thyroid carcinoma. Iranian Journal of Nuclear Medicine. 2008;16(30):8-15.

17. Bourgeois P. Scintigraphic investigations of the lymphatic system: the influence of injected volume and quantity of labeled colloidal tracer. Journal of nuclear medicine : official publication, Society of Nuclear Medicine. 2007;48(5):693-5.

18. Mariani G, Moresco L, Viale G, Villa G, Bagnasco M, Canavese G, et al. Radioguided sentinel lymph node biopsy in breast cancer surgery. Journal of nuclear medicine : official publication, Society of Nuclear Medicine. 2001;42(8):1198-215.

19. De Cicco C, Cremonesi M, Luini A, Bartolomei M, Grana C, Prisco G, et al. Lymphoscintigraphy and radioguided biopsy of the sentinel axillary node in breast cancer. Journal of nuclear medicine : official publication, Society of Nuclear Medicine. 1998;39(12):2080-4.

20. Safora Nikzad, Bijan Hashemi. MTT assay instead of the clonogenic assay in measuring the response of cells to ionizing radiation J Radiobiol. 2014;1(1):3-8.

21. Pijpers R, Meijer S, Hoekstra OS, Collet GJ, Comans EF, Boom RP, et al. Impact of lymphoscintigraphy on sentinel node identification with technetium-99m-colloidal albumin in breast cancer. Journal of nuclear medicine : official publication, Society of Nuclear Medicine. 1997;38(3):366-8.

22. Pelosi E, Bello M, Giors M, Ala A, Giani R, Bussone R, et al. Sentinel lymph node detection in patients with early-stage breast cancer: comparison of periareolar and subdermal/peritumoral injection techniques. Journal of nuclear medicine : official publication, Society of Nuclear Medicine. 2004;45(2):220-5.

23. Chakera AH, Friis E, Hesse U, Al-Suliman N, Zerahn B, Hesse B. Factors of importance for scintigraphic non-visualisation of sentinel nodes in breast cancer. European journal of nuclear medicine and molecular imaging. 2005;32(3):286-93.

24. Alavian-Ghavanini A, Stekovic S. Translational science: The future is already here. J Radiobiol. 2014;1(2):21-22.

25. Karimi-Shahri K. Neutron therapy: Radiation biology and treatment of cancers. J Radiobiol. 2014;1(2):38-39.

26. Nikzad S, Hashemi B, Mozdarani H, Hassan ZM. Comparison of various theoretical methods on the effect of intermittent irradiation schedule. J Radiobiol. 2014;1(2):23-27.

27. Schwartz GF, Meltzer AJ. Accuracy of axillary sentinel lymph node biopsy following neoadjuvant (induction) chemotherapy for carcinoma of the breast. The breast journal. 2003;9(5):374-9.

28. Knauer M, Konstantiniuk P, Haid A, Wenzl E, Riegler-Keil M, Postlberger S, et al. Multicentric breast cancer: a new indication for sentinel node biopsy--a multi-institutional validation study. Journal of clinical oncology : official journal of the American Society of Clinical Oncology. 2006;24(21):3374-80.

29. Statement by H.E. Mr. G. Ali Khoshroo, Deputy Foreign Minister for Legal and International Affairs, Second Session of the Prepcom for the 2005 NPT Review Conference, Apr. 29, 2003.

30. Nasri H. World Cancer Day 2014: "Increasing the Awareness". J Radiobiol. 2014;1(2):40-41. 